\title{
1 Tropical and Mediterranean biodiversity is disproportionately sensitive to land-use and
}

2 climate change

3

4 Tim Newbold ${ }^{1,},{ }^{*}$ Philippa Oppenheimer ${ }^{1}$, Adrienne Etard ${ }^{1}$, Jessica J. Williams ${ }^{1}$

5

$6 \quad{ }^{1}$ Centre for Biodiversity and Environment Research, Department of Genetics, Evolution and

7 Environment, University College London, Gower Street, London WC1E 6BT, United Kingdom

$8 \quad *$ Corresponding author: t.newbold@ucl.ac.uk

9

10 ORCIDs:

11 Tim Newbold: 0000-0001-7361-0051

12 Adrienne Etard: 0000-0002-1700-2972

13 Jessica Williams: $\underline{0000-0002-8275-7597}$

14

15 
Global biodiversity is undergoing rapid declines, driven in large part by changes to land use and climate. Global models help to understand the consequences of environmental changes for biodiversity, but tend to neglect important geographical variation in the sensitivity of biodiversity to these changes. Here we test whether biodiversity responses to climate change and land-use change differ among biomes (geographical units that have marked differences in environment and species composition). We find the strongest negative responses to both pressures in tropical biomes and in the Mediterranean. A further analysis points toward similar underlying drivers for regions where biodiversity losses are likely to be particularly large.

Keywords: biodiversity, biomes, climate change, global, land use, model

Introduction the sensitivity to each pressure: we find greater reductions in species richness in the most humandisturbed land uses and more negative predicted responses to climate change in areas of lower climatic seasonality, and in areas where a greater proportion of species are near their upper temperature limit. Within the land uses most heavily modified by humans, reductions in biodiversity were particularly large in regions where humans have come to dominate the land more recently. Our results will help to improve predictions of how biodiversity is likely to change with ongoing climatic and land-use changes, suggesting particularly large declines in the tropics where much future agricultural expansion is expected to occur. This finding could help to inform the development of the post-2020 biodiversity framework, by highlighting the under-studied

\section{Introduction}

Global biodiversity is continuing to decline despite increasing conservation efforts ${ }^{1-4}$. On the other hand, recent years have seen increases in people's awareness of the scale of biodiversity change and of the important roles that biodiversity plays, and also increases in funding for many aspects of conservation work and in the designation of protected areas ${ }^{5}$. It is likely that the failure of these 
increased conservation efforts to improve the state of biodiversity is caused by a continuing increase in the pressures on biodiversity ${ }^{5}$. There remain many gaps in our understanding of the effects of biodiversity pressures globally ${ }^{6}$, gaps which need to be addressed to understand better how to reduce the downward biodiversity trend.

Broad-scale models play a vital role in efforts to understand biodiversity change ${ }^{7,8}$, although they tend to focus on better-understood pressures ${ }^{8}$. Models can be used to attribute differences in biodiversity to putative pressures, generally pointing to large impacts of climate and land-use change $\mathrm{e}^{1,2,4,9,10}$. Currently, the available biodiversity data are patchy and biased ${ }^{11}$, and so models are necessary even to predict present-day biodiversity for many under-sampled species and locations. For exploring the future, biodiversity models can be applied to different alternative scenarios in an attempt to identify societal pathways that have a more positive outcome for biodiversity, 1,9,12,13.

Most broad-scale biodiversity models neglect important geographical variation in the sensitivity of biodiversity to human pressures. At the global scale, two main modelling approaches are used to capture the effects of land-use and climate change, both of which use spatial patterns to predict changes over time ('space-for-time substitution'). Responses of species to spatial variation in climate are typically represented using species distribution models, which use statistical methods to relate broad-scale data on a species' observed distributions to spatial variables describing the climate ${ }^{14}$ (and sometimes other aspects of the environment, including land use $\left.{ }^{10}\right)$. While species distribution models represent the response of each species individually, and thus implicitly capture geographical variation in sensitivity, it is rare to quantify that variation explicitly (although a recent study showed that tropical terrestrial communities have a smaller climate safety margin $\left.{ }^{15}\right)$. Globally, responses to land-use change have typically been assessed using statistical models that assess spatial differences in biodiversity across land-use types based on collations of fine-scale data ${ }^{1,16}$. These models generally assume that differences in biodiversity among land uses are constant across the whole 
terrestrial surface of the world ${ }^{1,16}$, although some models have considered tropical-temperate and taxonomic differences ${ }^{17,18}$. A recent study based on time-series data showed more negative biodiversity trends in tropical, temperate and Mediterranean biomes than in boreal areas or drylands ${ }^{19}$, highlighting important geographical differences in biodiversity change, likely driven in part by differences in the sensitivity of biodiversity to the major pressures.

There are several ecological and environmental differences among biomes that may cause geographical differences in the sensitivity of biodiversity. First, certain biomes (principally those in temperate areas) have been impacted by humans for a much longer period of time than others ${ }^{20}$. This long history of human use is likely to have already filtered out the most sensitive species even from natural habitats ${ }^{21}$, which is likely to lead to a smaller response of biodiversity to contemporary land-use change. Second, species have on average smaller range sizes in tropical biomes than elsewhere ${ }^{22}$. Smaller range size has been associated with a disproportionate sensitivity to both landuse change ${ }^{18}$ and climate change ${ }^{23}$. Third, there is a greater proportion of dietary and habitat specialist species found within tropical areas than in other biomes ${ }^{24}$. Dietary and habitat specialists have been shown to be most sensitive to both human land $u e^{25,26}$ and climate change ${ }^{27}$. Fourth, tropical species tend to have a slower 'pace of life', having smaller numbers of offspring and maturing more slowly than other species ${ }^{28}$. At least for birds, long generation time (which is associated with a slow pace of life) has been shown to confer greater sensitivity to human land use $\mathrm{e}^{25}$ and to climate change ${ }^{27}$. Fifth, the position of populations within species' geographical distributions and climatic niche limits (i.e. the observed climatic conditions that species inhabit) varies across biomes. Tropical biomes have a high proportion of populations living near the edge of their distribution and at the upper end of species' thermal limits, while high latitudes have a greater proportion of populations at lower thermal limits ${ }^{29}$. Similarly, species' upper and lower moisture limits will most often be reached in moist and dry biomes, respectively. In general, species near the edges of their geographical distribution have been shown to be more sensitive to environmental 
changes ${ }^{30}$. For bumblebees, recent climate change has been shown to impact most strongly those communities where a greater proportion of species are near the upper temperature limit of their observed distribution ${ }^{4}$. Land uses heavily modified by humans tend, on average, to be hotter and drier than natural habitats, which has led to shifts in ecological assemblages by favouring species associated with these climatic conditions ${ }^{31-33}$. The generally reduced canopy cover in heavily modified land uses means that there is also less of a buffering of temperature extremes compared to natural habitats ${ }^{34}$. Together, these differences in local climatic conditions in heavily modified land uses are favouring species that can tolerate greater extremes of climate (i.e., hotter maximum temperatures, colder minimum temperatures, and wetter and drier precipitation extremes) $31,32,35$. Finally, the very existence of biomes, with their widely differing vegetation structure, is caused by differences in climatic properties. In particular, tropical biomes have a lower degree of seasonality than temperate and high-latitude biomes. Species that have evolved to tolerate the narrower range of climatic and other environmental conditions within tropical biomes are likely to be more sensitive to environmental changes than those that evolved in areas with greater environmental variability ${ }^{36,37}$. Indeed, responses to both climate and land-use change have been shown to be most negative in areas of low climatic seasonality or among species inhabiting these areas 18,27,38. $^{2}$.

Here we ask whether sensitivity of biodiversity to climate and land-use change differs across biomes. We focus on species richness as a measure of biodiversity, which is easy to measure but captures only some of the many dimensions of biodiversity ${ }^{39}$ (in the Extended Data, we also present responses to land use of total community abundance and a measure based on the endemicity of species in the community). We further test the extent to which variation in the sensitivity of species richness is correlated with environmental and ecological differences among biomes that are hypothesised to influence sensitivity (climatic seasonality, average position of species within their climatic niche limits, and length of human land-use history). We hypothesise that tropical biomes will show the most negative responses because they harbour species known to be most sensitive on 
average to both pressures (slow-breeding specialists with small range sizes, which have evolved under more stable climatic conditions with a shorter history of land-use change), and because populations in tropical biomes tend to be nearer their upper thermal limits than elsewhere. Responses to land-use change may also be disproportionately negative in biomes with relatively drier climates because populations will tend to be closer to species' lower moisture limits.

To assess sensitivity to land-use change, we used mixed-effects models to compare species richness among land uses, using data from the PREDICTS database (Extended Data 1), which contains samples of communities from different land uses, mostly collected between 2000 and $2013^{40}$. Land uses considered were: primary vegetation (natural habitat, not known to have been destroyed in the past), secondary vegetation (natural habitat, recovering after being destroyed by human actions or extreme natural events), plantation forest (areas used to grow woody crops), cropland (areas used to grow herbaceous crops, including for livestock fodder), and pasture (areas used to graze livestock). In the final models, plantation forests and croplands were grouped into a single 'Harvested agriculture' category. The hierarchical structure of the land-use analysis means that differences in biodiversity among land uses are estimated within individual studies that span limited climatic gradients. Our estimates of likely sensitivity to climate change were based on a published ensemble of future projections of climate effects on species distributions ${ }^{9}$ between a 1960-1990 baseline and the future period 2061-2080 (assuming intermediate 'limited' dispersal ability; see Methods). We calculated sensitivity as the predicted change in grid-cell species richness per degree Celsius of expected mean temperature increase. The use of different input datasets and methods to estimate land-use and climate sensitivity was necessitated because we currently lack the data required to consider both pressures simultaneously ${ }^{9}$, and because climate and land use operate on biodiversity at very different scales. Land-use sensitivity was estimated using observed responses, whereas climate sensitivity was estimated based on future predicted changes because global datasets do not yet exist to allow a broad assessment of sensitivity to observed climate change. 
Nevertheless, for both pressures we express biodiversity changes in terms of species richness, so relative sensitivities across biomes should be comparable.

Results

Spatial differences in biodiversity among land uses varied strongly across biomes (Figure 1; Extended

Data 2). In secondary vegetation, species richness was more than $40 \%$ lower on average than in primary vegetation in the Mediterranean biome, around $10 \%$ lower in tropical forests and grasslands (although not significantly so in the latter case), and similar or even higher than in primary vegetation in temperate forests, temperate grasslands and drylands (Figure 1). In pastures, species richness was between 20 and $40 \%$ lower compared to primary vegetation in tropical forests and grasslands and in the Mediterranean biome, $17 \%$ lower in temperate grasslands, and similar or even higher than primary vegetation in temperate forest and drylands (Figure 1). Finally, for harvested croplands (woody plantations and herbaceous croplands), average species richness reductions were between 20 and 40\% for the tropical forest, temperate grassland and Mediterranean biomes, between 15 and $20 \%$ for tropical grasslands and temperate forests respectively, and around $10 \%$ for drylands (although not a significant reduction in the last case; Figure 1). Alternative groupings of either the land-use or biome classification did not markedly improve the fit of the models to the data (Extended Data 3 \& 4). Patterns were similar for two alternative measures of biodiversity: total community abundance and community-average range size (Extended Data 5 \& 6).

The sensitivity of vertebrate biodiversity to a $1^{\circ} \mathrm{C}$ increase in annual average temperature also varied widely across biomes (Figure 2; climate sensitivity was estimated by projecting biodiversity change against projected climate change between a 1960-1990 baseline and the future period 2061-2080, and then dividing by projected temperature change across the same time period). The most sensitive biomes were tropical forests, tropical grasslands and Mediterranean areas, with median 
projected local declines of between 10 and $13 \%$ in species richness for each degree of climate warming (Figure 2). Drylands showed intermediate sensitivity, with estimated declines of $5 \%$ of species for every degree increase in temperature. Finally, the biomes estimated to have the least sensitivity to climate change were temperate forests (median of $2 \%$ loss per degree of climate warming), temperate grasslands ( $1.5 \%$ loss) and boreal forests (1\% gain). Results were qualitatively very similar regardless of the emissions scenario assumed, but absolute sensitivities were less negative in most cases for the low-emissions (RCP 2.6) compared to the high-emissions (RCP 8.5) scenario: tropical forests $=-14 \%$; tropical grasslands $=-9.7 \%$; Mediterranean $=-6.1 \%$; Drylands $=-$ $2.8 \%$; temperate forests $=+1.4 \%$; temperate grasslands $=+1.4 \%$; boreal forests $=+7 \%$ (Extended Data 7).

Three biomes stood out for having large biodiversity reductions in land uses heavily modified by humans and a high sensitivity to climate warming: tropical forests, tropical grasslands and Mediterranean environments. These biomes showed an approximately $30 \%$ reduction in species richness in the most modified land uses compared to primary vegetation, and a $10-12 \%$ average local loss of species for each degree of climate warming (Figure 3). In contrast, the other biomes showed smaller sensitivities that were less consistent for land-use change and climate change (Figure 3).

Exploratory analyses suggested that both land-use and climate sensitivity may be associated with the same underlying factors (Table 1; Figure 4, where the sensitivity of biodiversity to land use for any given value of an explanatory variable is inferred as the relative species richness in disturbed land uses compared to the species richness in primary vegetation; Figure 5). The sensitivity of biodiversity to both climate change and to land use was greatest in areas with the lowest seasonality of both temperature and precipitation (Figure 4a, b; Figure 5a, b), and where a higher proportion of species were near the upper edge of their observed thermal niche (although for land use this was 
only true for harvested agriculture, not pasture; Figure 4c; Figure 5c). Contrary to our predictions, reductions in species richness in the most human-modified land uses (especially pastures) were greatest in areas where a higher proportion of species were near the centre of their precipitation niche (Figure 4d). As expected, responses to land use were strongest in areas that have experienced land-use impacts more recently (Figure 4e; this explanatory variable was not expected to have an effect on sensitivity to climate change, and so was not included in the analysis of climate responses). Although the explanatory variables helped to explain variation in sensitivity to land use among biomes, there remained a significant interaction between land use and biome in explaining species richness $\left(\chi^{2} 18,72=170, P<0.001\right)$. Owing to the need to use separate modelling paradigms for climate and land-use effects, we were not able to consider here any effects on biodiversity of interactions between land-use change and climate change, nor whether the effects of such interactions were strongest in certain biomes. This question should be addressed in future studies.

\section{Discussion}

\section{Our results demonstrate that species' sensitivity to climate and land-use change varies} geographically, and that high sensitivity to these two pressures coincides in the same parts of the world. The existence of such strong geographical variation implies that attempts to extrapolate inferences about the impact of climate and land-use change from well-studied areas s.4,41 $^{1,0}$ other parts of the world may be misleading. In particular, we find that tropical biomes (forests and grasslands) and the Mediterranean biome have a particularly high sensitivity to both pressures. We caution that quantitative comparisons of responses to climate change versus land-use change should not be made because of the very different scales at which these pressures operate, and because of the different methods used to assess their impacts. Nevertheless, the relative sensitivity of biomes to each pressure should be unaffected by the different methods used. Our results support previous studies that have shown greater reductions in biodiversity in heavily human-modified land uses in 
tropical compared to temperate areas ${ }^{17,18}$, but go further in showing differences among individual biomes. Our results are also consistent with a recent study showing similar geographical variation in historical biodiversity change using time-series data ${ }^{19}$.

The explanatory variables we considered help to explain geographical variation in the sensitivity of biodiversity, but do not completely explain the observed differences in biodiversity sensitivity among biomes. As expected, sensitivity to both pressures was highest in places with low climatic seasonality, probably because species confined to less-seasonal environments are not as resilient to environmental changes ${ }^{18,27,36}$. Sensitivity was also high in areas where a majority of species approach the hottest temperatures within their observed distributions (for land use this was true for harvested agriculture, but not for pastures used for livestock grazing). The disproportionate biodiversity reductions in agriculture in areas dominated by species near their upper temperature limits probably results from the fact that heavily human-modified land uses tend to have hotter maximum temperatures than natural habitats ${ }^{42}$. The results with regard to precipitation were more equivocal. Unexpectedly, there were greater biodiversity reductions in the most modified land uses where there were more species approaching the centre of their precipitation niche (Figure 4). Precipitation patterns, and resulting changes in moisture availability, are not predicted to change as consistently and monotonically as temperature either with regional climate change $\mathrm{e}^{43}$ or with human land use $\mathrm{e}^{33,44}$. As expected, the effects of human land use were strongest in areas that have experienced a relatively short history of land-use disturbance. This is consistent with suggestions that a longer period of disturbance filters out the most sensitive species from ecological communities rendering the remaining communities less sensitive to further disturbance ${ }^{21}$.

Future efforts to model broad-scale biodiversity changes should consider geographical variation in the sensitivity of biodiversity. Previous models, especially those focusing on the effects of land-use change, have generally ignored geographical variation in sensitivity altogether ${ }^{1,9,16}$. The existence of 
wide differences among regions in the sensitivity of biodiversity to environmental changes has important implications for predictions of future biodiversity. Tropical biomes are predicted to experience most future agricultural expansion ${ }^{45}$, and to be among the first areas to experience unprecedented temperatures as a result of climate chang $\mathrm{e}^{46}$. The disproportionate sensitivity of tropical biodiversity that we find thus implies that future biodiversity changes may be larger than suggested by most previous models.

The disproportionate sensitivity of tropical and Mediterranean biomes points to important gaps in our understanding of the ecology and conservation of these areas ${ }^{47}$. Data on biodiversity are patchy, and often show strong bias toward temperate forests and grasslands ${ }^{11,19}$. Our results suggest that the under-studied biodiversity of tropical biomes is most sensitive to major environmental changes, supporting previous research that has highlighted the sensitivity of tropical biodiversity to land-use change ${ }^{17,18}$. Tropical and Mediterranean areas are the richest in overall numbers of species ${ }^{48}$ and in numbers of endemic species ${ }^{49}$, and so higher proportional losses of species in these biomes will translate into even larger losses in absolute terms. We need more research to understand better the causes and consequences of the sensitivity of tropical and Mediterranean biodiversity. The growing availability of databases describing changes in biodiversity over time will likely allow progress in this area, although such databases still under-represent tropical areas ${ }^{50}$.

Broad-scale models of the response of biodiversity to both land-use and climate change have a number of important known limitations. Both model types ignore important ecological effects such as biotic interactions and adaptation to environmental changes ${ }^{51}$, and synthetic models of land-use impacts may generally underestimate sensitivity because of the difficulty of establishing baseline conditions without human influence ${ }^{52}$. To affect the qualitative patterns of biodiversity sensitivities across biomes, any model artefacts would have to affect disproportionately certain biomes over others. This may be true for some of the ecological limitations of the models. For example, biotic 
interactions are thought to be relatively more important in shaping biodiversity in tropical than temperate latitudes ${ }^{53}$. A failure to account for biotic interactions and other factors limiting species' distributions will influence the inference of climatic niche limits, which determine expected responses to climate change in the models. Unfortunately, data on true physiological limits are available for too few species to include in an analysis such as is presented here ${ }^{35}$, but future studies should explore this question further. Our results suggest an important role of climatic variation in explaining the observed sensitivity of biomes. However, in the correlative analysis we present here, we cannot rule out the possibility that climate determines the distribution of biomes, but that some other feature of biomes (for example, vegetation differences or differences in species composition unrelated to climate) determines sensitivity. Data limitations are another potential source of bias, if data quantity or quality differ markedly across biomes. For assessing climate impacts, we used globally consistent data on the extent of species ranges ${ }^{54,55}$, rather than opportunistically-collected data that tend to be geographically biased ${ }^{11}$. Nevertheless, differences in the accuracy of these range estimates across biomes are very likely. Similarly, the data used to assess responses to land use $^{40}$ were compiled to be as geographically representative as possible, and biomes were sampled roughly in proportion to their area ${ }^{56}$. Yet, as with the data on species' ranges, we cannot rule out geographical variation in data quality. We focus in this study on species richness as a biodiversity measure that can be estimated by both the modelling approaches we use, while acknowledging that species richness cannot capture the many dimensions of biodiversity ${ }^{39}$. We show that similar patterns in land-use responses are obtained for two other measures based on abundance or species endemicity (Extended Data $5 \& 6$ ), but future modelling work should aim to represent a broader suite of biodiversity metrics. Finally, it was not possible to isolate completely observed responses to climate change and land-use change, given that both pressures are operating within complex realworld ecological systems. While the hierarchical structure of the models of land-use responses, with differences in biodiversity fitted within individual studies that span relatively small climatic 
gradients, should factor out most effects of regional climate change, climate inevitably plays a role in shaping observed responses (indeed, we show this to be the case in our analyses).

In conclusion, we have shown that biodiversity in tropical and Mediterranean biomes is disproportionately sensitive to both land-use and climate change, which has important implications for conservation in these areas. Further work is needed to incorporate differences in sensitivity into broad-scale models of biodiversity changes. Moreover, results such as these can contribute to the discussions around the post-2020 biodiversity framework ${ }^{57}$, by highlighting the disparity in how environmental changes impact biodiversity in different geographic regions of the world and showing a disproportionate sensitivity of the under-studied tropics. Importantly, our results suggest that the high level of land-use and climate change expected in tropical biomes in the coming decades will affect ecological communities that are particularly sensitive to these environmental changes, and thus biodiversity declines may be stronger than previous models have implied.

\section{Methods}

\section{Defining biomes}

There are several schemes by which the world can be divided into biogeographical units, differing in the set of species they contain ${ }^{58-60}$. A recent global study showed marked changes in species composition across the boundaries of ecoregions $s^{60}$, which are a nested sub-unit of biomes ${ }^{61}$. For understanding large-scale differences in the sensitivity of biodiversity to environmental changes, a coarser division into biomes is more practical than an ecoregion-based division, given the paucity of biodiversity data for most ecoregions ${ }^{11,40}$. 
We derived estimates of the spatial distribution of biomes from The Nature Conservancy's map of global ecoregions ${ }^{62}$. This map divides the world into 16 different biomes, 11 of which we considered in this study: Tropical and Subtropical Moist Broadleaf Forests; Tropical and Subtropical Dry Broadleaf Forests; Tropical and Subtropical Coniferous Forests; Temperate Broadleaf and Mixed Forests; Temperate Conifer Forests; Boreal Forests/Taiga; Tropical and Subtropical Grasslands, Savannas and Shrublands; Temperate Grasslands, Savannas and Shrublands; Montane Grasslands and Shrublands; Mediterranean Forests, Woodlands and Scrub, and Deserts and Xeric Shrublands. Biomes excluded owing to a paucity of biodiversity data were: Tundra; Mangroves; Flooded Grasslands and Savannas; Inland Water; and Rock and Ice. Although a newer version of this map is available $^{61}$, it is unlikely that its use would have made a noticeable difference to the broad-scale under future climate scenarios (see below).

Framework for modelling biodiversity responses

We used mixed-effects models to estimate differences in biodiversity among different land-use types, and species distribution models to project likely biodiversity responses to climate change. Treating the effects of land use and climate in separate models is often necessitated by the fact that these pressures operate at very different spatial scales, and because the data documenting responses to the two pressures don't transcend the different scales (data on species distributions are typically not accurate enough to capture effects of land use, while data on the effects of land use do not span large enough climatic gradients to capture the effects of regional climate change) $)^{9}$. In some regions, distribution and land-use data are sufficiently accurately resolved that it is possible to 
include the effects of land use directly into species distribution models ${ }^{10}$. Alternatively, for some well-known taxonomic groups, expert knowledge can be used to predict species' responses to landuse change in species distribution models ${ }^{64}$. For global multi-clade analyses, however, it remains necessary to use separate modelling paradigms ${ }^{9}$. The difference in methods used means that the absolute estimates of responses to each pressure are not directly comparable. Nevertheless, the relative sensitivities of biodiversity to each pressure across biomes should be unaffected. All input datasets are detailed in Extended Data 8.

\section{Estimating biodiversity response to land use}

We obtained data describing differences in biodiversity among land-use types from the database of the PREDICTS Project ${ }^{63}$. This database is a global collation of published comparisons of ecological assemblages across different types of land use, both natural and human-dominated ${ }^{56}$. We excluded any studies that focused on a single species, because these cannot be expected to give a reliable estimate of species richness ${ }^{1}$. Original samples were collected in the field between 1984 and 2013 (95\% of locations were sampled since 2000). The PREDICTS database is structured such that data from each published Source may be divided into one or more Studies, distinguished if the data were collected using a different sampling protocol. Studies may be divided into one or more Spatial Blocks, within which one or more distinct Sites are sampled. The data for each Site consists of a list of taxa, in most cases with recorded abundances, but sometimes just simple presence or absence (a very small number of records give the overall species richness of a group of species). The predominant land use at each site was classified based on the description of the sampled habitat as given in the original source publication, as follows ${ }^{56}$ : primary vegetation describes natural habitat with no record of historical destruction of the vegetation (including remnant patches in urban areas); secondary vegetation describes natural habitat that is known to have been destroyed historically, either by human actions or extreme natural events, but which is now recovering to its 
natural state; plantation forests are areas used for cultivation of woody crops (such as fruit, oil-palm, coffee or timber plantations); cropland is land used for cultivating herbaceous crops (including fodder for livestock); pastures are areas regularly or permanently used for livestock grazing; and urban areas are those used for human settlements or civic amenity, or areas where the vegetation has been transformed for human recreation. We excluded urban land use in this study, because there were too few urban samples to allow a consideration of biome differences. The PREDICTS database contains data for 47,044 species of vertebrates, invertebrates, plants and fungi ${ }^{40}$. The dataset analysed in this study were from 20,585 sites in 11 out of 14 of the world's terrestrial biomes (the tundra, flooded grasslands and mangroves biomes were discarded from the analysis because they were represented by too few sites) and from 91 of the world's countries.

We used mixed-effects models ${ }^{65}$ to fit differences in sampled species richness as a function of land use and biome. We modelled species richness using generalised linear mixed-effects models with a Poisson distribution of errors. We included a random intercept of study identity to account for the differences in sampling protocols among studies, and to ensure that comparisons were made within studies that spanned relatively small environmental gradients, thus excluding most effects of climate change on observed biodiversity differences ${ }^{1}$. We additionally included a random intercept of site identity (i.e. an observation-level random intercept), to account for the over-dispersion present ${ }^{66}$. Some combinations of land use and biome are poorly sampled, so using the finest divisions of these variables (i.e. all 11 biomes and all 5 land uses) would reduce the potential generality of our findings. Therefore, we initially sought the most parsimonious groupings of land use and biome for explaining observed differences in species richness. For example, croplands and pastures could be grouped together as a single 'agriculture' class, or tropical conifer forest, tropical moist broadleaf forest and tropical dry broadleaf forest could be grouped as 'tropical forest'. Using the finest division of biomes, we compared different groupings of land use. Separately, using the finest division of land uses, we compared different groupings of biome. For all four sets of models, we selected the one with the 
lowest AIC value as the best-fitting. We then fit a final model of species richness using the combination of the best-fitting land-use grouping and the best-fitting biome grouping. This procedure allowed us to maximise the generality of our results, without unduly sacrificing explanatory power. We focus in the main text on changes in species richness, which can be measured more-or-less consistently with respect to both land-use change and climate change. However, species richness does not capture all facets of biodiversity ${ }^{39}$. To test whether the estimated sensitivity of biodiversity to land-use change was influenced by choice of biodiversity metric, we repeated the models also for the total sampled abundance of each community ${ }^{1}$, and the average range size of species within the community (weighted by species' abundance) ${ }^{18}$. The latter metric captures the degree of endemicity of species within each community, and so may be a more sensitive metric of the effects of land use ${ }^{18}$. We estimated the average range size of species within each community using published estimates already calculated for the communities sampled in the PREDICTS database (DOI: 10.6084/m9.figshare.7262732.v1) ${ }^{18}$. These estimates were originally made ${ }^{18}$ by: 1) taking all records for each species from the Global Biodiversity Information Facility (GBIF) database; 2) mapping these records onto a 110-km equal-area (Behrmann projection) grid; 3) summing the total area of the occupied grid cells (which should be relatively insensitive to geographical outliers among the biodiversity records); and 4) calculating the average area-ofoccupancy across all species within any sampled community in the PREDICTS database, weighted by species' abundance (see ref. 18 for full details). We assumed that the GBIF records would be adequate for estimating broad relative differences in species' area of occupancy (indeed, previous analyses have shown that effects of these range size estimates on responses to land use are relatively robust to alternative methods of calculation ${ }^{18}$ ).

\section{Estimating biodiversity sensitivity to climate change}


We obtained our estimates of the predicted response of biodiversity (vertebrate assemblage species richness) to climate change from published projections based on species distribution models ${ }^{9}$. These projections were derived using species' distribution data for 20,938 terrestrial vertebrate species from published extent-of-occurrence range maps ${ }^{54,55}$. While such maps tend to underestimate the full extent of species' ranges while overestimating local occupancy ${ }^{67}$, they are the best data with which to capture broad-scale responses to climate change of a large number of species ${ }^{9}$. The published distribution models ${ }^{9}$ used an ensemble of five modelling algorithms (DOMAIN, BIOCLIM, Maxent, Generalised Linear Models and Random Forests), fit using the dismo package Version 1.1-4 in R. For any given location (10-km grid cell) we took the median of the projections across the ensemble. Each range map was converted to a raster at $10-\mathrm{km}$ spatial resolution, using a cylindrical equal-area projection. Each occupied cell was considered a presence record in the distribution models. The Maxent algorithm drew 10,000 background points from all grid cells (occupied and unoccupied) within realm-biome combinations containing at least one presence record. For the Generalised Linear Models and Random Forests, pseudo-absence records were drawn at random from unoccupied cells within realm-biome combinations that also contain a presence record. The distribution of each species was modelled as a function of four climatic variables shown to have good explanatory power for vertebrate distributions: minimum temperature of the coldest month, total annual precipitation, growing degree days and water balance. Distribution models were evaluated against a reserved $20 \%$ of records, using the Area Under the Receiver Operating Characteristic Curve (AUC) statistic; all models with an AUC $>0.8$ were retained. The distribution models were projected onto current and future climate estimates under the Representative 450 Concentration Pathways (RCP) scenarios ${ }^{68}$ derived from the Worldclim Version 1.4 database.

451 Continuous predictions from all models were converted to a binary prediction of presence or 452 absence using a threshold that minimizes the difference between model sensitivity and specificity. In the original paper in which these projections were presented ${ }^{9}$, three dispersal scenarios were used: no dispersal, unlimited dispersal, and intermediate 'limited' dispersal. In this study, we focused on 
the projections under 'limited' dispersal. Birds and mammals were assumed to be able to expand their ranges by $3 \mathrm{~km}$ per year, and reptiles and amphibians by $0.5 \mathrm{~km}$ per year (see ref. 9 for full details of the distribution modelling and projection methods). To estimate the sensitivity of ecological communities to climate change across space, here we took the future projections (from a 1960-1990 baseline until the period 2061-2080) of percentage change in the number of species present in each location (negative for species loss, positive for species gain) divided by the number of degrees by which annual average temperature is expected to increase within the same timeframe and under the same scenario (negative for projected decreases in temperature). We obtained the latter estimates of change in annual mean temperature also from the WorldClim Version 1.4 database. Because biodiversity may show a non-linear response to climate warming, we tested the robustness of the results to using two different RCP climate scenarios with varying overall projected temperature increase: the RCP2.6 scenario, which is characterised by strong climate-mitigation efforts, and RCP8.5, which represents a high-emissions scenario.

Explaining biome differences in biodiversity sensitivity to land use and climate change

In order to attempt to explain any observed differences in biodiversity sensitivity among biomes, we considered a set of factors that differ among biomes and that have been hypothesised or shown to influence differences in biodiversity among land uses or the response of species to climate change: climate (temperature and precipitation) seasonality; the community-average position of species with respect to their observed climatic (temperature and precipitation) niche limits; and the number of years since the landscape was substantially impacted by human land-use activities. We initially considered two other variables (the average proportion of habitat specialists within a community; and the average range-size of species within a community). However, these variables were strongly correlated with others and can be less reliably measured across the breadth of species considered in this study. 
Temperature and precipitation seasonality estimates were derived directly from WorldClim Version $1.4^{69}$ (bioclimatic variables, Bio4 and Bio15, respectively) at 10-arc-minute spatial resolution.

Thermal and precipitation position indices were estimates of the community-average position of species within their observed (i.e. realised) thermal and precipitation niches (i.e. a value of 0 would indicate that all species were at their lower thermal or precipitation niche limits, i.e. cold and dry limits, respectively; whereas a value of 1 would indicate that all species were at their hot or wet niche limits). For each vertebrate species (species that have relatively reliable information on broadscale distribution), we estimated position within niche limits based on the minimum and maximum conditions experienced throughout its distribution. Estimates of species distributions were the extent-of-occurrence maps as described above. We did not use GBIF data (see above) to estimate position within species' thermal and precipitation niches, because the patchy and biased nature of the GBIF data ${ }^{11}$ means that estimates of the precise position of range (and thus climatic niche) boundaries are likely to be unreliable. We processed the distribution maps by: 1) excluding areas where species are considered to be vagrant, or present only during migration; 2) excluding areas outside the known elevational limits for the species; and 3) resampling the maps to a 10-km cylindrical equal-area (Behrmann) spatial projection. The grain size of $10 \mathrm{~km}$ was selected as a reasonable trade-off between precision and map accuracy. We defined the limits of a species thermal niche as the minimum across its distribution of the minimum temperature of the coldest month (bioclimatic variable 'bio6' from WorldClim Version $1.4^{69}$ ) and the maximum across its distribution of the maximum temperature of the warmest month ('bio5'). For precipitation, we defined niche limits as the minimum across a species' distribution of the total precipitation in the driest month ('bio14') and the maximum across the distribution of the total precipitation in the wettest month ('bio13'). For a given location, we then calculated a species' thermal or precipitation position as the average across the year of the monthly average maximum temperature or monthly 
total precipitation values - also obtained from WorldClim Version $1.4^{69}$ - after rescaling the raw

508

509

510

511

512

513

514 climatic variables to take values of 0 and 1 at species' estimated niche limits. Finally, for every location (10-km grid cells), we estimated the community-average thermal and precipitation position indices as the average of the species-level values for all species potentially occurring within a grid cell, with potential occurrence defined using the same distribution maps as before. We resampled all climatic variables to the same $10-\mathrm{km}$ equal-area projection as the distribution maps.

Our estimates of the length of time that a landscape had been substantially impacted by human land-use activities was based on the HYDE reconstruction of historical land use ${ }^{45}$. Specifically, we calculated the number of years since each $0.5^{\circ}$ grid cell is estimated to have first surpassed $30 \%$ conversion of natural habitat to human-dominated uses (croplands, pastures and urban environments). A 30\% threshold has been suggested previously to represent a level at which significant effects of fragmentation will begin to be felt for some species ${ }^{70}$, and the time since this level of conversion occurred has been shown previously to explain responses of biodiversity to land use $^{1}$.

To test the importance of these factors in explaining land-use responses, we fit more complex mixed-effects models of species richness as a function not only of land use and biome interactions, but also of land use in interaction with the additional explanatory variables. We fit the additional explanatory variables and their interactions with land use as fixed effects in the model, allowing quadratic terms for the continuous explanatory variables because we hypothesised that the responses would likely be non-linear. The random-effects structures were the same as for the earlier models of land-use responses: site identity nested within study identity. We used backward stepwise model selection to exclude non-significant terms (likelihood-ratio test, $\alpha=0.05$ ). 
To explain geographical variation in the sensitivity of vertebrate assemblages to climate change, we modelled the estimates of percent species richness change per degree of temperature change (see above) as a function of the same explanatory variables as used for analysing land-use responses. We initially fit these models using ordinary least squares regression, allowing only linear terms for the continuous variables, because over-fitting was likely with such a large sample (all terrestrial 10-km grid cells). Since species richness, species richness change and all of the explanatory variables are likely to show strong, positive spatial autocorrelation, we repeated all of the models using spatial autoregression (using the lagsarlm function in the spdep package Version $1.1-3^{71}$ in $\mathrm{R}$ ).

\section{Author Contributions}

542

T.N. and P.O conceived and designed the study and carried out the main analyses. A.E. and J.J.W. input analytical tools and important insight on aspects of the work. T.N. wrote the final manuscript, with substantial inputs from all authors.

546

\section{Data availability}

548

All data required to run the analyses are published on FigShare:

http://doi.org/10.6084/m9.figshare.12674372.

551

\section{Code availability}

554 All code used in the analyses is publicly available at:

555 https://github.com/timnewbold/BiomeSpecificResponsesPublic. 
The authors declare no competing interests.

560

\section{Acknowledgments}

562

563

This work was supported by a Royal Society University Research Fellowship to T.N. J.J.W. and A.E. (RPG-2015-073).

567

\section{References}

569

570

1. Newbold, T. et al. Global effects of land use on local terrestrial biodiversity. Nature $\mathbf{5 2 0}, \mathbf{4 5 -}$ 50 (2015).

2. WWF. Living Planet Report - 2018: Aiming Higher (eds Grooten, M. \& Almond, R. E. A.). (WWF, 2018).

3. Hallmann, C. A. et al. More than 75 percent decline over 27 years in total flying insect biomass in protected areas. PLoS One 12, e0185809 (2017).

4. Soroye, P., Newbold, T. \& Kerr, J. Climate change contributes to widespread declines among bumble bees across continents. Science 367, 685-688 (2020).

5. Tittensor, D. P. et al. A mid-term analysis of progress toward international biodiversity targets. Science 346, 241-244 (2014).

6. Newbold, T. et al. Climate and land-use change homogenise terrestrial biodiversity, with consequences for ecosystem functioning and human well-being. Emerg. Top. Life Sci. 3, 207219 (2019). 
7. Nicholson, E. et al. Scenarios and models to support global conservation targets. Trends Ecol. Evol. 34, 57-68 (2019).

8. IPBES. The Methodological Assessment Report on Scenarios and Models of Biodiversity and Ecosystem Services (eds Ferrier, S., Ninan, K. N., Leadley, P., Alkemade, R., Acosta, L. A., Akçakaya, H. R., Brotons, L., Cheung, W. W. L., Christensen, V., Harhash, K. A., KabuboMariara, J., Lundquist, C., Obersteiner, M., Pereira, H. M., Peterson, G., Pichs-Madruga, R., Ravindranath, N., Rondinini, C. \& Wintle, B. A.). (Secretariat of the Intergovernmental Science-Policy Platform on Biodiversity and Ecosystem Services, 2016).

9. Newbold, T. Future effects of climate and land-use change on terrestrial vertebrate community diversity under different scenarios. Proc. R. Soc. B Biol. Sci. 285, 20180792 (2018).

10. Marshall, L. et al. The interplay of climate and land use change affects the distribution of EU bumblebees. Glob. Chang. Biol. 24, 101-116 (2018).

11. Meyer, C., Kreft, H., Guralnick, R. \& Jetz, W. Global priorities for an effective information basis of biodiversity distributions. Nat. Commun. 6, 8221 (2015).

12. Visconti, P. et al. Projecting global biodiversity indicators under future development scenarios. Conserv. Lett. 9, 5-13 (2016).

13. Mace, G. M. et al. Aiming higher to bend the curve of biodiversity loss. Nat. Sustain. 1, 448451 (2018).

14. Araújo, M. B., Alagador, D., Cabeza, M., Nogués-Bravo, D. \& Thuiller, W. Climate change threatens European conservation areas. Ecol. Lett. 14, 484-492 (2011).

15. Pinsky, M. L., Eikeset, A. M., McCauley, D. J., Payne, J. L. \& Sunday, J. M. Greater vulnerability to warming of marine versus terrestrial ectotherms. Nature 569, 108-111 (2019).

16. Alkemade, R. et al. GLOBIO3: a framework to investigate options for reducing global terrestrial biodiversity loss. Ecosystems 12, 374-390 (2009).

17. Martins, I. S. \& Pereira, H. M. Improving extinction projections across scales and habitats using the countryside species-area relationship. Sci. Rep. 7, 12899 (2017). 
18. Newbold, T. et al. Widespread winners and narrow-ranged losers: land use homogenizes biodiversity in local assemblages worldwide. PLoS Biol. 16, e2006841 (2018).

19. Blowes, S. A. et al. The geography of biodiversity change in marine and terrestrial assemblages. Science 366, 339-345 (2019).

20. Klein Goldewijk, K., Beusen, A., Van Drecht, G. \& De Vos, M. The HYDE 3.1 spatially explicit database of human-induced global land-use change over the past 12,000 years. Glob. Ecol. Biogeogr. 20, 73-86 (2011).

21. Balmford, A. Extinction filters and current resilience: the significance of past selection pressures for conservation biology. Trends Ecol. Evol. 11, 193-196 (1996).

22. Stevens, G. C. The latitudinal gradient in geographic range: how so many species coexist in the tropics. Am. Nat. 133, 240-256 (1989).

23. Thuiller, W., Lavorel, S. \& Araújo, M. B. Niche properties and geographical extent as predictors of species sensitivity to climate change. Glob. Ecol. Biogeogr. 14, 347-357 (2005).

24. Forister, M. L. et al. The global distribution of diet breadth in insect herbivores. Proc. Natl. Acad. Sci. U. S. A. 112, 442-447 (2015).

25. Newbold, T. et al. Ecological traits affect the response of tropical forest bird species to landuse intensity. Proc. R. Soc. London Ser. B Biol. Sci. 280, 20122131 (2013).

26. Rader, R., Bartomeus, I., Tylianakis, J. M. \& Laliberté, E. The winners and losers of land use intensification: pollinator community disassembly is non-random and alters functional diversity. Divers. Distrib. 20, 908-917 (2014).

27. Pacifici, M. et al. Species' traits influenced their response to recent climate change. Nat. Clim. Chang. 7, 205-208 (2017).

28. Wiersma, P., Munoz-Garcia, A., Walker, A. \& Williams, J. B. Tropical birds have a slow pace of life. Proc. Natl. Acad. Sci. 104, 9340-9345 (2007).

29. Sunday, J. M. et al. Thermal-safety margins and the necessity of thermoregulatory behavior across latitude and elevation. Proc. Natl. Acad. Sci. U. S. A. 111, 5610-5615 (2014). 
30. Orme, C. D. L. et al. Distance to range edge determines sensitivity to deforestation. Nat. Ecol. Evol. 3, 886-891 (2019).

31. Frishkoff, L. O., Hadly, E. A. \& Daily, G. C. Thermal niche predicts tolerance to habitat conversion in tropical amphibians and reptiles. Glob. Chang. Biol. 21, 3901-3916 (2015).

32. Frishkoff, L. O. et al. Climate change and habitat conversion favour the same species. Ecol. Lett. 19, 1081-1090 (2016).

33. Williams, J. J. \& Newbold, T. Local climatic changes affect biodiversity responses to land use: A review. Divers. Distrib. 26, 76-92 (2020).

34. De Frenne, P. et al. Global buffering of temperatures under forest canopies. Nat. Ecol. Evol. 3, 744-749 (2019).

35. Williams, J. J., Bates, A. E. \& Newbold, T. Human-dominated land uses favour species affiliated with more extreme climates, especially in the tropics. Ecography 43, 391-405 (2020).

36. Janzen, D. H. Why mountain passes are higher in the tropics. Am. Nat. 101, 233-249 (967).

37. Srinivasan, U., Elsen, P. R. \& Wilcove, D. S. Annual temperature variation influences the vulnerability of montane bird communities to land-use change. Ecography 42, 1-11 (2019).

38. Newbold, T. et al. Global patterns of terrestrial assemblage turnover within and among land uses. Ecography 39, 1151-1163 (2016).

39. Hillebrand, H. et al. Biodiversity change is uncoupled from species richness trends: consequences for conservation and monitoring. J. Appl. Ecol. 55, 169-184 (2018).

40. Hudson, L. N. et al. The database of the PREDICTS (Projecting Responses of Ecological Diversity In Changing Terrestrial Systems) project. Ecol. Evol. 7, 145-188 (2017).

41. Chen, I.-C., Hill, J. K., Ohlemuller, R., Roy, D. B. \& Thomas, C. D. Rapid range shifts of species associated with high levels of climate warming. Science 333, 1024-1026 (2011).

42. Senior, R. A., Hill, J. K., González del Pliego, P., Goode, L. K. \& Edwards, D. P. A pantropical analysis of the impacts of forest degradation and conversion on local temperature. Ecol. Evol. 
43. Trenberth, K. E. Changes in precipitation with climate change. Clim. Res. 47, 123-138 (2011).

44. Fu, B., Wang, J., Chen, L. \& Qiu, Y. The effects of land use on soil moisture variation in the Danangou catchment of the Loess Plateau, China. Catena 54, 197-213 (2003).

45. Hurtt, G. C. et al. Harmonization of land-use scenarios for the period 1500-2100: 600 years of global gridded annual land-use transitions, wood harvest, and resulting secondary lands. Clim. Change 109, 117-161 (2011).

46. Mora, C. et al. The projected timing of climate departure from recent variability. Nature 502, 183-187 (2013).

47. García-Vega, D. \& Newbold, T. Assessing the effects of land use on biodiversity in the world's drylands and Mediterranean environments. Biodivers. Conserv. 29, 393-408 (2020).

48. Jenkins, C. N., Pimm, S. L. \& Joppa, L. N. Global patterns of terrestrial vertebrate diversity and conservation. Proc. Natl. Acad. Sci. 110, E2602-E2610 (2013).

49. Myers, N., Mittermeier, R. A., Mittermeier, C. G., da Fonseca, G. A. B. \& Kent, J. Biodiversity hotspots for conservation priorities. Nature 403, 853-858 (2000).

50. Dornelas, M. et al. BioTIME: A database of biodiversity time series for the Anthropocene. Glob. Ecol. Biogeogr. 27, 760-786 (2018).

51. Pearson, R. G. \& Dawson, T. P. Predicting the impacts of climate change on the distribution of species: are bioclimate envelope models useful? Glob. Ecol. Biogeogr. 12, 361-371 (2003).

52. Newbold, T., Sanchez-Ortiz, K., De Palma, A., Hill, S. L. L. \& Purvis, A. Reply to 'The biodiversity intactness index may underestimate losses'. Nat. Ecol. Evol. 3, 864-865 (2019).

53. Roslin, T. et al. Higher predation risk for insect prey at low latitudes and elevations. Science 356, 742-744 (2017).

54. IUCN. The IUCN Red List of Threatened Species. Version 2013.7. http://www.iucnredlist.org/ (2013).

55. Birdlife International \& NatureServe. Bird Species Distribution Maps of the World. Version 2.0. 
http://www.birdlife.org/datazone/info/spcdownload (2012).

56. Hudson, L. N. et al. The PREDICTS database: a global database of how local terrestrial biodiversity responds to human impacts. Ecol. Evol. 4, 4701-4735 (2014).

57. Convention on Biological Diversity. Zero Draft of the Post-2020 Global Biodiversity Framework. (Convention on Biological Diversity, Resolution CBD/WG2020/2/3, 2020).

58. Holt, B. G. et al. An update of Wallace's zoogeographic regions of the world. Science 339, 7478 (2013).

59. Kissling, W. D., Sekercioglu, C. H. \& Jetz, W. Bird dietary guild richness across latitudes, environments and biogeographic regions. Glob. Ecol. Biogeogr. 21, 328-340 (2012).

60. Smith, J. R. et al. A global test of ecoregions. Nat. Ecol. Evol. 2, 1889-1896 (2018).

61. Dinerstein, E. et al. An ecoregion-based approach to protecting half the terrestrial realm. Bioscience 67, 534-545 (2017).

62. The Nature Conservancy. Terrestrial Ecoregions of the World. http://maps.tnc.org/gis_data.html (2009).

63. Hudson, L. N. et al. Dataset: The 2016 Release of the PREDICTS Database. (2016) doi:10.5519/0066354.

64. Powers, R. P. \& Jetz, W. Global habitat loss and extinction risk of terrestrial vertebrates under future land-use-change scenarios. Nat. Clim. Chang. 9, 323-329 (2019).

65. Bolker, B. M. et al. Generalized linear mixed models: a practical guide for ecology and evolution. Trends Ecol. Evol. 24, 127-135 (2008).

66. Rigby, R. A., Stasinopoulos, D. M. \& Akantziliotou, C. A framework for modelling overdispersed count data, including the Poisson-shifted generalized inverse Gaussian distribution. Comput. Stat. Data Anal. 53, 381-393 (2008).

67. Herkt, K. M. B., Skidmore, A. K. \& Fahr, J. Macroecological conclusions based on IUCN expert maps: a call for caution. Glob. Ecol. Biogeogr. 26, 930-941 (2017).

68. van Vuuren, D. P. et al. The representative concentration pathways: an overview. Clim. 
69. Hijmans, R. J., Cameron, S. E., Parra, J. L., Jones, P. G. \& Jarvis, A. Very high resolution interpolated climate surfaces for global land areas. Int. J. Climatol. 25, 1965-1978 (2005).

70. Andrén, H. Effects of habitat fragmentation on birds and mammals in landscapes with different proportions of suitable habitat: a review. Oikos 71, 355-366 (1994). spatial association. TEST 27, 716-748 (2018).

Figure Legends

Figure 1. Differences in species richness among land-use types, across different biomes: a) Tropical forest; b) Tropical grasslands; c) Drylands; d) Mediterranean; e) Temperate forest; and f) Temperate grassland. Plots show the percentage change in species richness compared to primary vegetation (PV), in secondary vegetation (SV), pasture (PAS) and areas of harvested agriculture (woody plantations and herbaceous croplands; HARV). Error bars show $95 \%$ confidence intervals. Sample sizes at the bottom of each panel refer to the number of sites in each combination of land use and biome. The most complex models were the best fitting - including the finest division of both biome and land use, and their interaction (Extended Data 3 \& 4). However, because relatively few sites were sampled in some biomes and thus to maximise the number of biomes for which we could make a reasonably confident inference of land-use responses, we show here the results based on a coarser division of both biome and land use. The model with coarser land-use and biome groupings still showed a relatively similar fit to the data compared to the most complex models (Species richness: $\mathrm{R}^{2}$ conditional $=$ $0.61, R^{2}$ marginal $\left.=0.025\right)$.

Figure 2. Predicted sensitivity of biodiversity to climate change across biomes. Shown is the predicted percentage change in vertebrate species richness for each ${ }^{\circ} \mathrm{C}$ of climate warming expected under the RCP 8.5 scenario. Results were qualitatively very similar under the lower-emissions RCP2.6 scenario (Extended Data 7). 
grasslands (Trop G, $n_{\text {cells }}=203,690$ ), drylands (Dry, $n_{\text {cells }}=279,178$ ), Mediterranean (Med, $n_{\text {cells }}=31,630$ ),

temperate forest (Temp F, $\mathrm{n}_{\text {cells }}=170,680$ ), temperate grasslands (Temp $\left.G, n_{\text {cells }}=144,369\right)$ and boreal forest (Bor $\left.\mathrm{F}, \mathrm{n}_{\text {cells }}=168,005\right)$. Thick horizontal black lines show median values across all grid cells within the biome, boxes extend to the first and third quartiles, and whiskers to $1.5 \times$ the inter-quartile range.

Figure 3. Relationship across biomes between climate and land-use sensitivity. We estimate climate sensitivity as the predicted percentage species richness change of the average ecological assemblage under $1^{\circ} \mathrm{C}$ of warming under the RCP 8.5 scenario. We estimated land-use sensitivity as the average percentage difference in species richness between human-dominated land uses (plantation forests, cropland and pasture) and primary vegetation.

Figure 4. Patterns of species richness among land-use types moderated by putative explanatory variables. variable: a) temperature seasonality, b) precipitation seasonality, c) average proximity of species in an assemblage to lower (value $=0.0$ ) or upper (value $=1.0$ ) monthly temperature limits based on species' observed distributions; d) average proximity of species in an assemblage to lower or upper maximum precipitation limits based on species' observed distributions; e) the number of years since the landscape became at least $30 \%$ converted to human-dominated land uses. All interactions between land use and the explanatory variables had a significant association with species richness $\left(\chi^{2}>23, P<0.001\right.$; Table 1$)$. Sensitivity at a given value of an explanatory variable is inferred as the relative reduction in species richness in disturbed land uses compared with the species richness in primary vegetation.

Figure 5. Relationships of predicted biodiversity sensitivity to climate change with putative explanatory variables. a) temperature seasonality; b) precipitation seasonality, c) average proximity of species in an assemblage to lower (value $=0.0$ ) or upper (value $=1.0$ ) monthly temperature limits based on species' observed distributions; d) average proximity of species in an assemblage to lower or upper maximum precipitation limits based on species' observed distributions. We estimated climate sensitivity as the percentage change in species richness for each ${ }^{\circ} \mathrm{C}$ of warming expected under the RCP 8.5 climate scenario. 
768 Relationships are shown here for a random sample of 10,000 10-km grid cells from across the world's

769 terrestrial surface. We derived fitted relationships (shown by red lines) from a simple linear model relating

770 climate sensitivity to each variable, but the direction and significance of relationships was consistent when

771 using a spatial autoregressive model. 
Table 1. Statistics for the ability of potential explanatory variables to explain responses of species richness

to land use. Shown are the statistics (Chi-squared values, $\chi^{2}$; degrees of freedom, DF; and P values from

likelihood-ratio tests) describing the effect on species richness of interactions between the explanatory variables and land use (using the most parsimonious classification, highlighted in bold in Extended Data 3).

\begin{tabular}{|l|l|l|l|}
\hline Interaction with land use & $\chi^{2}$ & DF & $P$ \\
\hline Biome & 170 & 18,70 & $<0.001$ \\
\hline Temperature seasonality & 23.8 & 6,70 & $<0.001$ \\
\hline Precipitation seasonality & 67.3 & 6,70 & $<0.001$ \\
\hline Thermal position & 70.3 & 6,70 & $<0.001$ \\
\hline Precipitation position & 203.5 & 6,70 & $<0.001$ \\
\hline Time since landscape & 25.8 & 6,70 & $<0.001$ \\
conversion to human- & & & \\
\hline dominated land uses & & & \\
\hline
\end{tabular}

779 